\title{
The impact of atmospheric mountain lee waves on systematic geodetic errors observed using the Global Positioning System
}

\author{
Seiichi Shimada ${ }^{1}$, Hiromu Seko ${ }^{2}$, Hajime Nakamura ${ }^{2}$, Kazumasa Aonashi $^{2}$, and Thomas A. Herring ${ }^{3}$ \\ ${ }^{1}$ National Research Institute for Earth Science and Disaster Prevention, 3-1 Tennodai, Tsukuba, Ibaraki 305-0006, Japan \\ ${ }^{2}$ Meteorological Research Institute, 1-1 Nagamine, Tsukuba, Ibaraki 305-0052, Japan \\ ${ }^{3}$ Department of Earth, Atmospheric and Planetary Sciences, Massachusetts Institute of Technology, \\ 77 Massachusetts Ave., Cambridge, Massachusetts 02139, U.S.A.
}

(Received November 22, 2001; Revised February 4, 2002; Accepted February 4, 2002)

\begin{abstract}
Atmospheric mountain lee waves excited by a strong westerly wind ahead of approaching cold front are shown to have significant effect on GPS positioning. Before the approach of the cold front significant atmospheric gradients caused by the inhomogeneous water vapor are detected at the sites along the east coast of the Izu Peninsula because of a wet atmosphere to the west of these sites. In contrast, the island site $6 \mathrm{~km}$ east of the coast detects a strong gradient with the opposite sense. The Geostationary Meteorological Satellite cloud shows rows of the clouds due to mountain lee waves consistent with the GPS measurements. A numerical simulation explains the mountain lee waves. The atmospheric perturbations induce large systematic errors in the estimates of horizontal positions of the sites in the region.
\end{abstract}

\section{Introduction}

The northern part of the Izu Peninsula is subject to frequent seismic swarms associated with the magma activity (Shimada et al., 1990; Okada and Yamamoto, 1991; Aoki et al., 1999) where the northern tip of the Philippine Sea Plate collides with the central part of Honshu Island. For monitoring the crustal movements associated with these seismic swarms, a dense Global Positioning System (GPS) array is deployed in this region with inter-site spacing of three to ten $\mathrm{km}$. Between the 1st and 11th of March 1997, one of such seismic swarm occurred in this area. More than $110 \mathrm{~mm}$ horizontal displacement is observed at the GPS site near the epicentral region as well as up to $50 \mathrm{~mm}$ vertical motion (Geographical Survey Institute, 1997).

In the midst of the activity a cold front passed through the region and the inhomogeneity of the water vapor seems to bring significant systematic errors in the estimates of the GPS site positions. Estimation of horizontal gradients in the disturbed atmosphere has been successfully applied to improve the repeatability of the estimate coordinates (McMillan, 1995; Chen and Herring, 1997; Bar-Sever et al., 1998). We have also applied the technique to estimate the horizontal gradient in this paper. However, in our case, although the estimated gradients are consistent with the meteorological inferences about the state of the atmosphere, that is, eastward dry along the east coast of the peninsula and westward dry at the Hatsushima Island about $7 \mathrm{~km}$ off the east coast, there persist apparent large errors in some of the estimates of the site horizontal positions in the region.

Copy right(C) The Society of Geomagnetism and Earth, Planetary and Space Sciences (SGEPSS); The Seismological Society of Japan; The Volcanological Society of Japan; The Geodetic Society of Japan; The Japanese Society for Planetary Sciences.
In this region, $\mathrm{N}-\mathrm{S}$ oriented mountains run along the east coast facing the Sagami Bay, whereas to the west of the mountains there is a flat plane abutting Suruga Bay. Thus the mountain lee waves occur in the region when there is strong wind, almost perpendicular to the orientation of the mountains, associated with the approach of the cold front. Hooke (1986) gives an analytic solution for mountain lee waves generated by a solitary mountain with Gaussian distribution. His solution suggests that in the Izu case mountain lee waves will undulate to east of the mountains and that there will be non-linear inhomogeneity in the troposphere over the GPS sites. In our analysis applying the estimation of the atmospheric gradient may show the limitation of this approximation.

\section{Observations and Analysis}

On 7th March 1997 (day of year 66), a strong west wind blew in the region ahead of the approach of the cold front (Fig. 1). Actually the west wind blew from 9:00 UT on 6th to $15: 00 \mathrm{UT}$ on 7 th with more than $10 \mathrm{~m} / \mathrm{sec}$ measured from 0:00 UT to 11:00 UT on 7th at the Ajiro site (4111 site in Fig. 3). Because the west wind is almost perpendicular to the N-S oriented mountains; atmospheric mountain waves were excited on the leeward side of the mountains. The Geostationary Meteorological Satellite (GMS) observed the cloud rows of the mountain lee waves (three to five lines detectable) in the visible light cloud images of 23:36 UT on 6th, 0:37 UT, and 1:36 UT on 7th (Fig. 2). Judging from the cloud rows of the mountain lee waves, the clouds are on the top of the mountain line and over the sea about $10 \mathrm{~km}$ off the coast. Although the 2:36 UT data is not available, the weak undulations seen in the 3:36 UT cloud image, suggests the lee waves had largely dissipated by this time. 


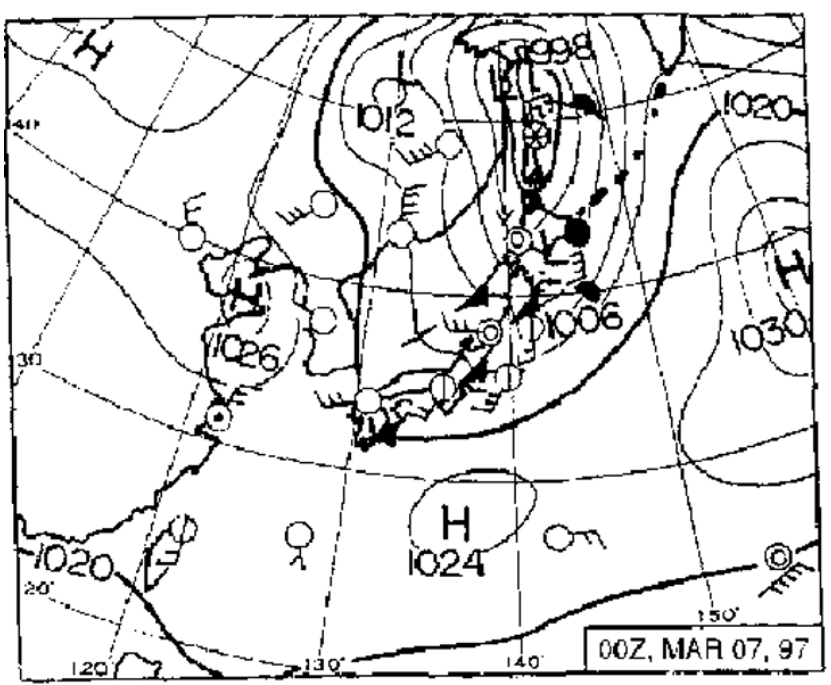

Fig. 1. The Synoptic weather chart around Japan at 00 UTC on 7th March 1997. A cold front is passing Japanese Islands. In the studied area, the front passes between 12-15 UTC based on the analysis of surface meteorological observation data
In the GPS data analysis, we used the 18 local sites in and around the Izu Peninsula and 7 global sites (Fairbanks in Alaska, Kokee Park in Hawaii, Guam in the Western Pacific, Shanghai in China, Taejon in Korea, Usuda and Tsukuba in Japan) from the International GPS Service (IGS) network sites. The IGS final orbit ephemeris was fixed in the analysis. The coordinates of the 18 local sites, as well as the coordinates of the 7 global sites, were fixed at values determined from the analysis of years of data before and after the date. The uncertainty of these coordinates is less than $3 \mathrm{~mm}$. However, positions of the two sites near the swarm epicentral area (KWN, 2107 and 2108 in Fig. 3) are affected by the swarm activity and their positions can change by up to $10 \mathrm{~mm}$ per day. We determined the coordinates of these two sites from the next day data (8th March) when mountain waves were not observed. The difference between the 6th and 8th March coordinates is 10-20 mm for those three sites. The coordinates of all sites were fixed to those values to avoid the trade-off between the estimations of the coordinates of the vertical component and the zenith delay. For the global sites, the coordinates and velocity determined by International Earth Rotation Service (IERS) were used (IERS Terrestrial Reference Frame 1997 (Boucher et al., 1998)).
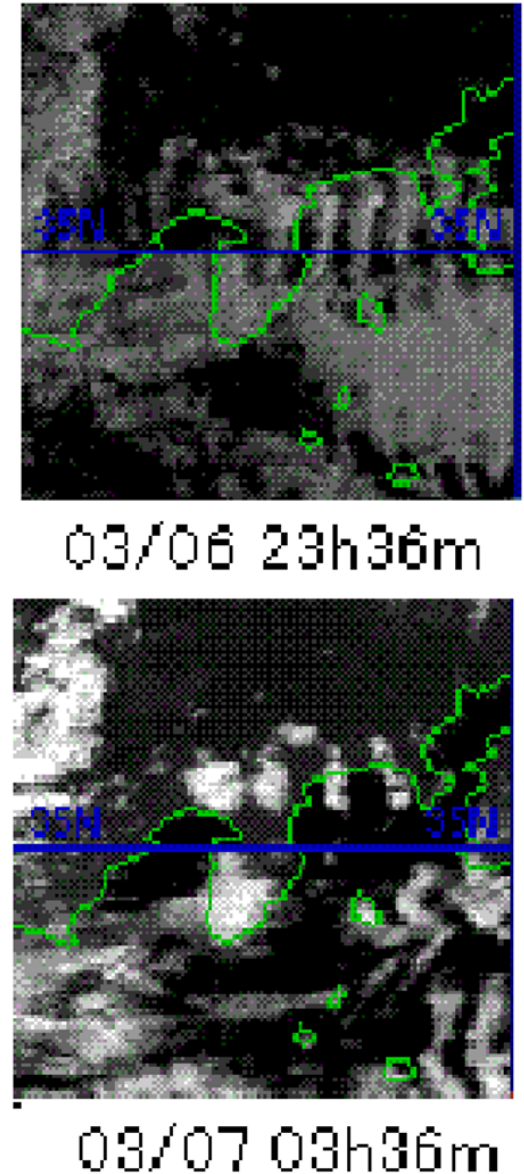
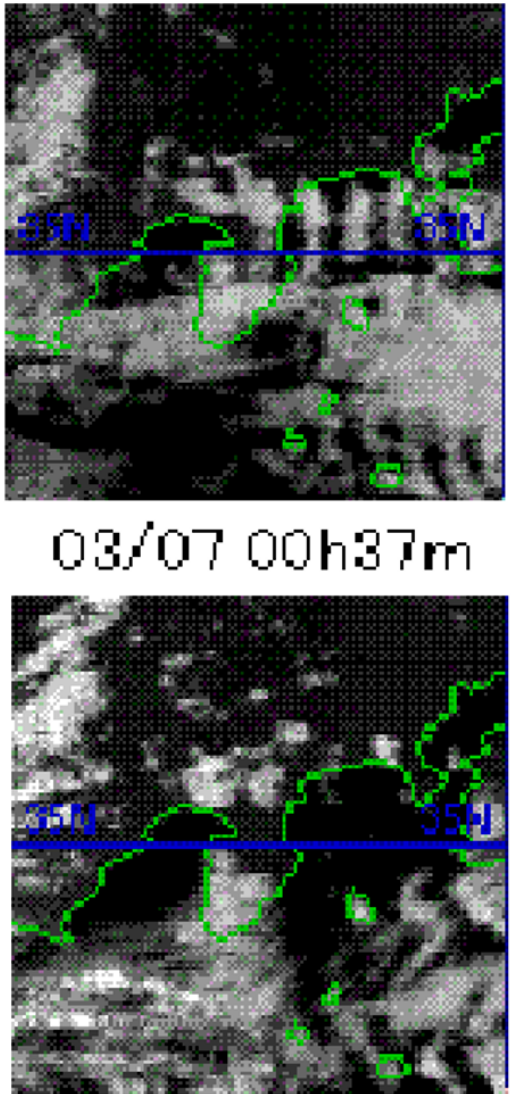

$03 / 0704 h 30 \mathrm{~m}$
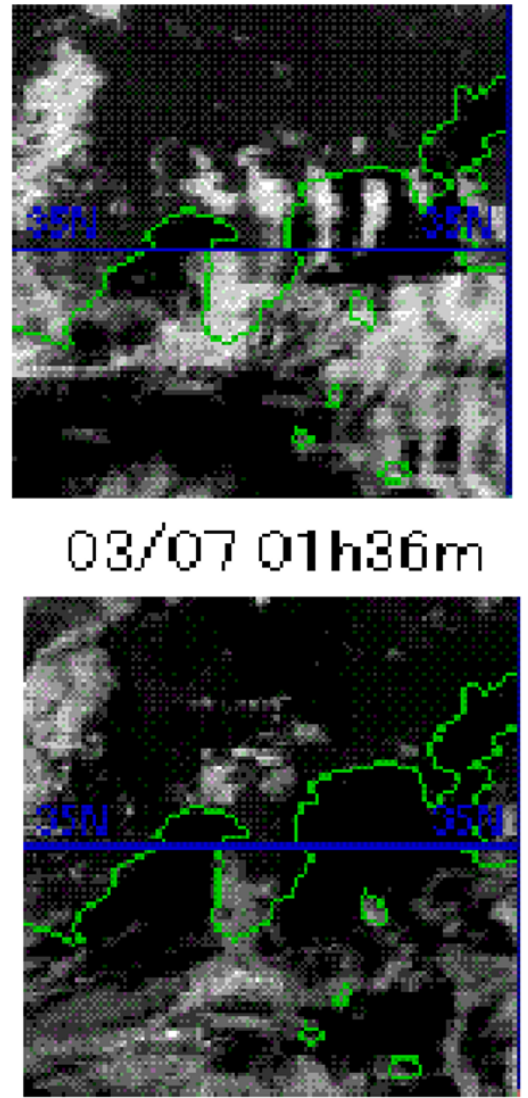

$03 / 07$ o5h36m

Fig. 2. The visible cloud images taken by the Geostationary Meteorological Satellite (GMS) in the region of this study in Central Japan showing cloud rows due to the mountain lee waves along the east coast of the northern part of the Izu Peninsula and on the sea in the Sagami Bay east of the peninsula. 


\section{7/03/07 00:00-24:00UT HORIZONTAL GRADIENTS}

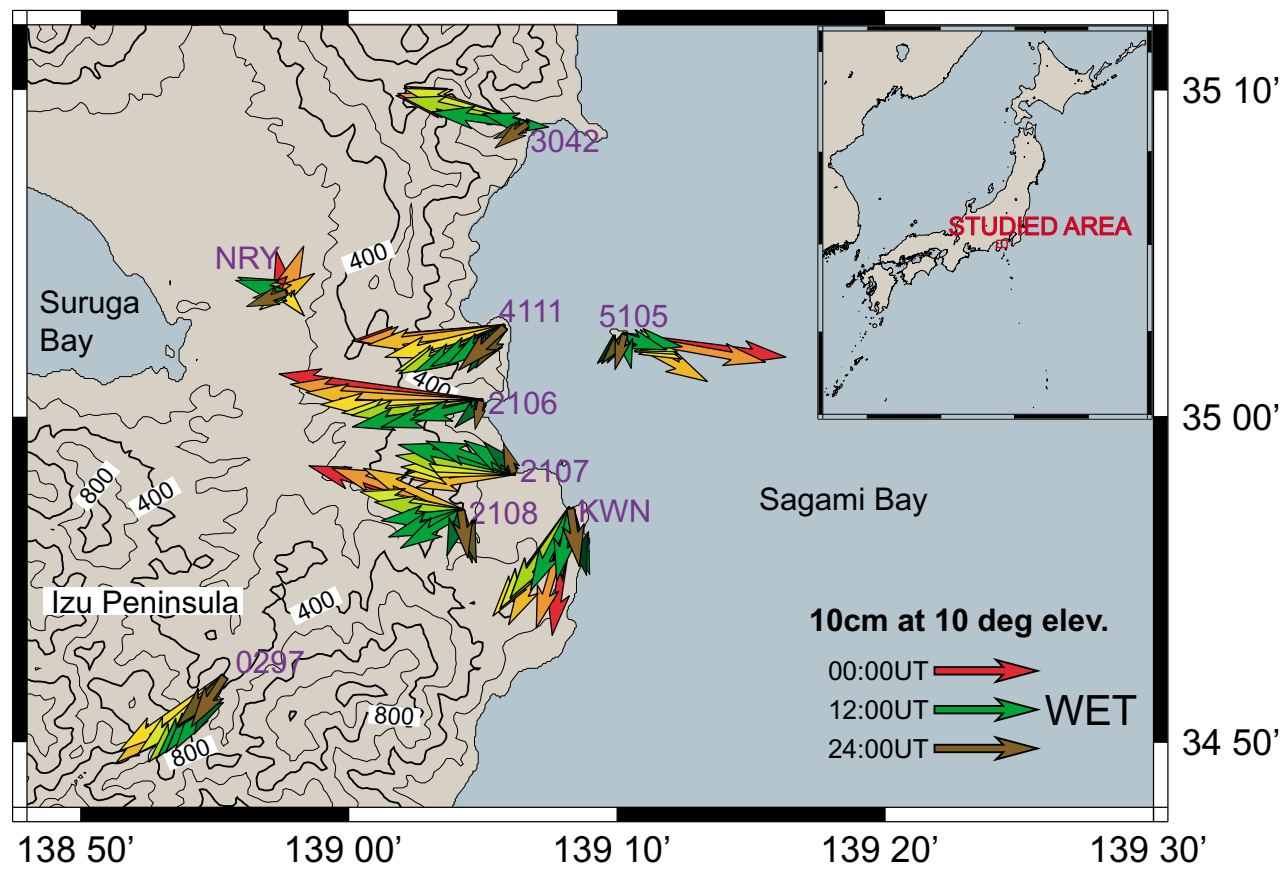

Fig. 3. Two-hourly estimates of horizontal gradients on 7th March 1997. The gradient vectors of the sites along the east coast of the Izu Peninsula indicates westward wet and cold, while that of the site 5105 , about $7 \mathrm{~km}$ of the east coast, shows eastward wet and cold. Thus the narrow zone east of the coast from 3042 to 2107 seems to be dry and warm due to the mountain lee waves.

The estimated parameters are the zenith delays at each site, modeled as piecewise linear functions with points every 30 minutes and horizontal gradients (Chen and Herring, 1997) at each site with points every 2 hours. We also estimated and fixed independent carrier phase ambiguities. The elevation angle cutoff is ten degree, although the observed cutoff angle of the GEONET sites (all sites except KWN site in the area) is 15 degree. We used the GAMIT program (King and Bock, 2000) in the analysis. The global sites were included in the analysis to allow the estimates of the absolute values of atmospheric delay parameters (as opposed to the relative values between sites).

\section{Results}

Figure 3 shows the horizontal gradient solutions for the stations in the northern part of the Izu Peninsula on 7 th March with topography contours overlaid. The sites between 3042 and 2107, along the east coast where mountains run parallel to the coastline, show atmospheric gradient vectors directed significantly westward between 0 and 12 UTC with amplitudes that decrease during the period. The site 5105 , on the small island named 'Hatsushima' about $7 \mathrm{~km}$ off the east coast, shows the opposite behavior especially between 0 and 4 UTC when the mountain lee waves are most significant in the GMS cloud images. The gradients of the KWN site south of the coastline of the peninsula are consistent with the fact that the atmosphere above the sea east of the east coast is relatively dry and probably warm between 0 and 12 UTC, consistent with the expectation from the GMS cloud images mentioned above. The NRY site west of the mountains seems to have no significant gradients during the period. NRY site is located on the windward side of the mountains and should not be affected by the mountain waves. The site 0297 in the mountain of the Central Izu Peninsula shows SW directed gradient, probably due to the mountain lee waves excited by the deviated wind among the mountain area.

\section{Discussion}

Using the JMA/MRI (Meteorological Research Institute) non-hydrostatic model (NHM) (Saito, 1997) with the horizontal grid interval of $250 \mathrm{~m}$, we simulated the mountain lee waves and tried to interpret their role in the atmospheric delay. Number of grids is $300 \times 300$ for horizontal and 38 for vertical component. Figure 4(a) shows the model zenith wet delay (proportional to precipitable water vapor). $X$ - and $Y$ axes is distance from the grid origin. We can reproduce the mountain lee waves consisted with the mountain lee waves observed in Fig. 2, although the wave length is a little shorter than observation in the midst and eastern part of the Sagami Bay. Since there are no upper air observation in the model area, we give the vertical profile of temperature observed by Hamamatsu, about $130 \mathrm{~km}$ WSW from the field, and wind observed by Tateno, about $200 \mathrm{~km}$ NE from the field, for the initial western boundary condition of the model $(X=0 \mathrm{~km})$ uniformly, to fit the GMS cloud images using the trial and error for the data of the nearby three radiosonde sites concerning on the vertical profile of temperature and wind.

Figure 4(b) shows the vertical profile of the wet zenith delay per $\mathrm{km}$. The numerical model is consistent with the atmospheric gradient estimated by GPS analysis in that the dry air is between the east coast of the peninsula and the 
(a) Map of Wet Zenith Delay (cm)

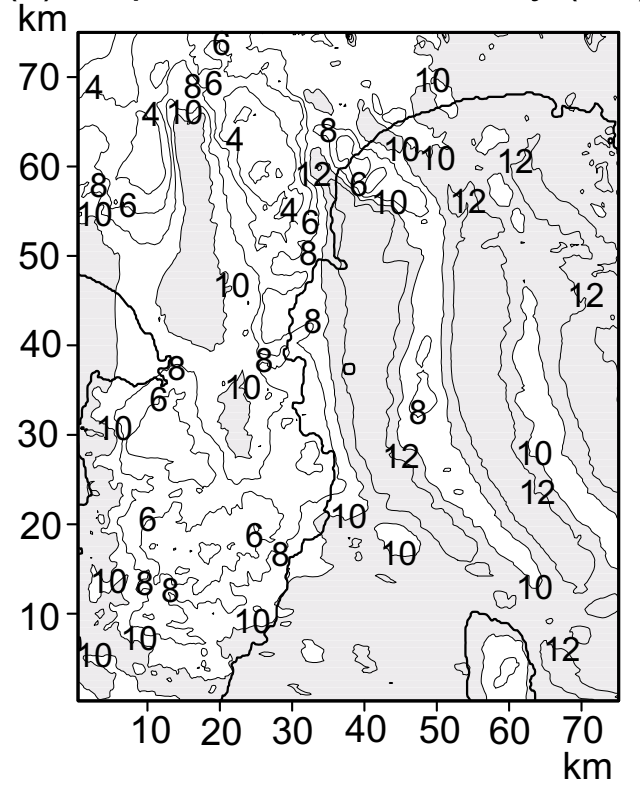

(b) Wet Delay $(\mathrm{cm})$ per $\mathrm{km}$ along $\mathrm{y}=37.5 \mathrm{~km}$ $\mathrm{km}$

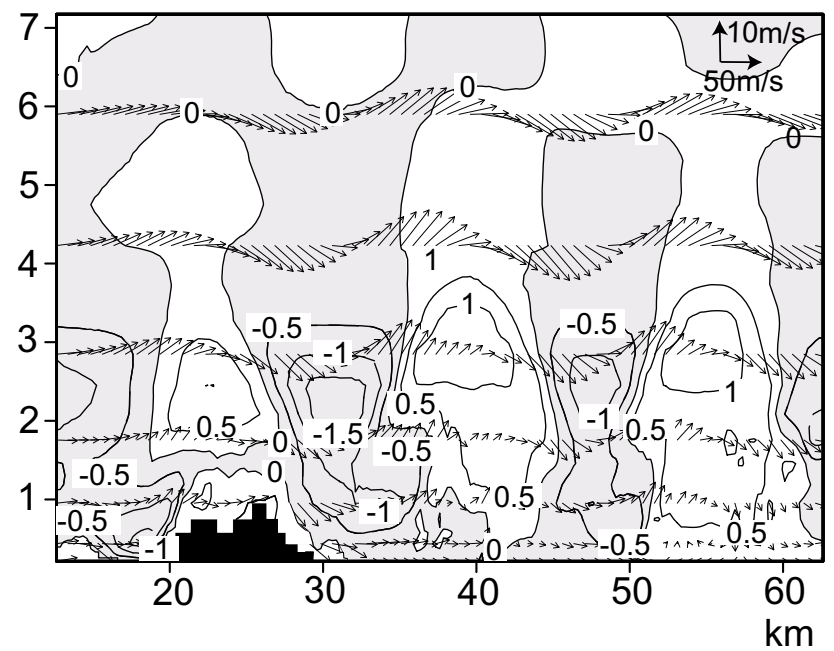

Fig. 4. (a) The map of the numerical model zenith wet delay (proportional to the precipitable water vapor) when standing waves become stable. Mountain along the east coast of the northern Izu Peninsula causes mountain waves on the leeward side of the mountains with a wavelength of about $20 \mathrm{~km}$ parallel to the mountain line. The mountain lee waves are standing waves and hence there is no specific time associated with this figure. (b) The vertical profile of the wet zenith delay per $\mathrm{km}$ in the E-W axis across near Ajiro and Hatsushima sites with the wind vectors. Vertical winds are four times larger than horizontal ones for the exaggeration.

Hatsushima Island. You can find the mountain lee waves make obviously non-linear horizontal anomaly, which is different from the hypothesis of horizontal simple gradient troposphere model of the GPS analyzing software.

If an air mass is forced to lift along a mountain slope, it expands adiabatically resulting in lowering its temperature with cooling rate of 10 degree $/ \mathrm{km}$ if no condensation occurs. Since the air temperature decreases upward by about 6.5 degree $/ \mathrm{km}$ in general, the temperature of the air mass forced to lift along the mountain slope will become lower than the surrounding air. After the air mass passed over the summit of the mountain, it will descend because there is no supporting force like a mountain slope and that the air mass is heavier than the surrounding air. The air mass goes downward passing a level where the temperature of the air mass is the same as that of the surrounding air. After entering the lighter surrounding air, the air mass receives a force to accelerate its upward motion and then the motion of the air mass changes from downward to upward. The consequence is the oscillation of the air mass in the vertical direction. During the air mass is moving horizontally by the prevailing surrounding air flow, the combination of the horizontal and vertical motions results in a wave pattern in the downstream of the mountain.

If there is a wavy flow pattern in the vertical like a mountain wave, an air mass, which moves on this flow experiences upward and downward motion. In general an air near the ground surface contains more water vapor than the upper air. Consider an air mass near the surface, which has abundant water vapor. When the air mass moves upward in the upward flow area in a mountain wave, then water vapor is transported to a higher level. This means the increase of the top of moist layer. The maximum height of the moist layer will be at the level where vertical motion is zero. (It is not correct that water vapor is large in the vertical motion area as stated in this paper because the situation is complex compared with a simple model.) On the other hand, during the air mass experiences the downward motion, the top of the moist layer decreases.

There is another important point to comment. There should be horizontal convergence of air to support upward air flow and divergence for downward flow. The convergence means the concentration of water vapor and divergence sweeping away of water vapor. This effect is also contributed to the positive anomalies at the upward motion area and negative at downward motion area.

The horizontal site coordinates estimates from the GPS analysis on 7th March using the GAMIT software, show large systematic errors up to $14 \mathrm{~mm}$ (Fig. 5) from the difference of site coordinate solutions of (March 7)-(March 6) and (March 8)-(March 7). The vectors of the systematic error are mostly directed opposite to the observed atmospheric horizontal vectors, thus the vectors are opposite direction with about same amplitudes between (March 7)-(March 6) and (March 8)-(March 7). No significant systematic errors are found in the solutions on 8th March, although small systematic errors caused by the approach of the cold front may contaminate on 6 th. The most likely explanation for these deviations is that the atmospheric horizontal gradient, as well as the observed atmospheric horizontal vectors, causes the large systematic errors in the estimates of the horizontal positions. When we analyze the same day including the gradient estimations, there still exist some systematic errors in the estimated site positions, although some of the errors are significantly reduced. For the sites KWN, 2107 and 2108 some part of the motions is possibly the real crustal movements caused by the seismic swarm activity. The systematic errors still remaining in the solution with the gradient estimations are possibly due to the mountain lee waves on 6th 

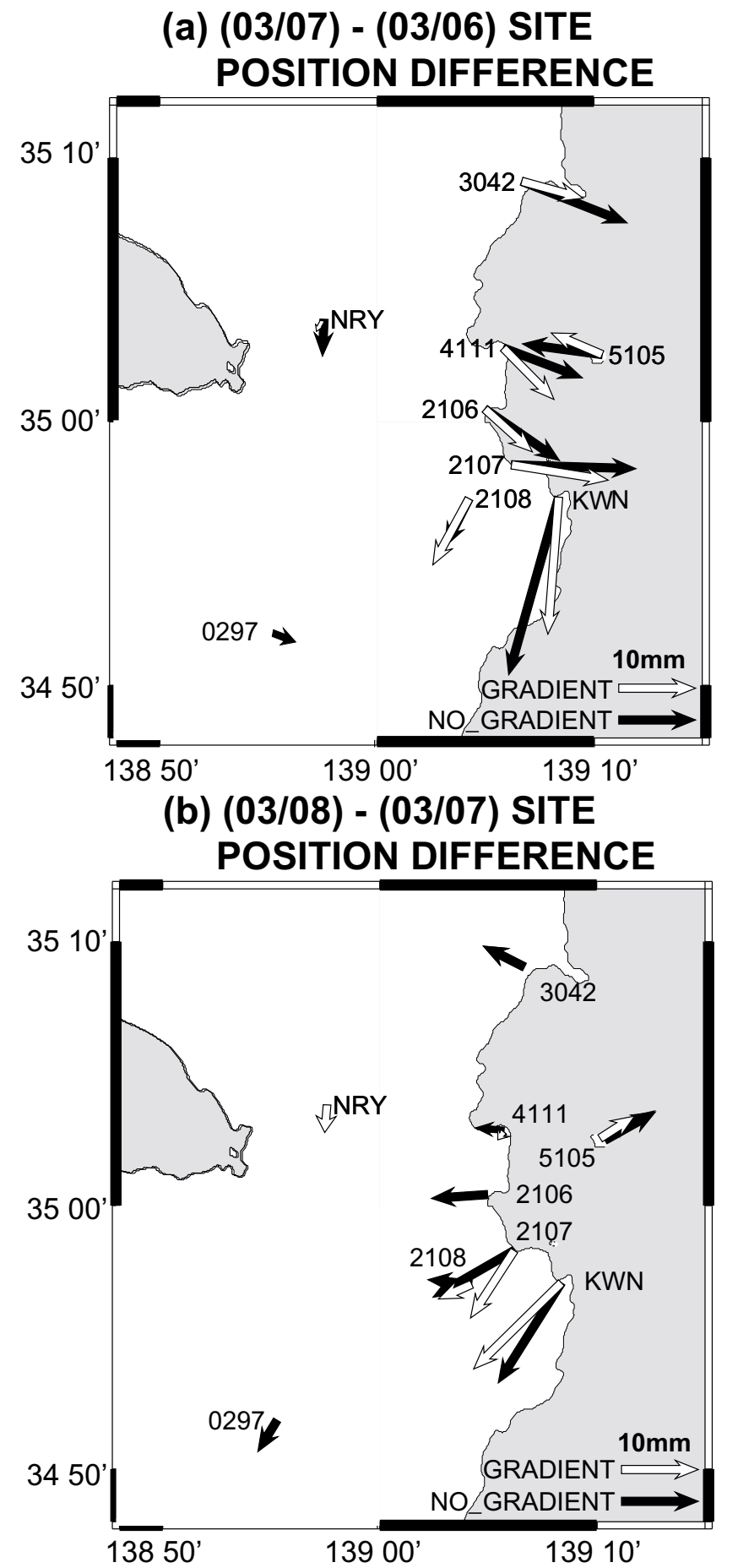

Fig. 5. Difference of daily solution of site coordinates. Difference of site coordinate solutions of (a) (March 7)-(March 6) and (b) (March 8)-(March 7) are opposite direction in the most sites, suggesting large systematic errors in the solution of 7th March in the case of no atmospheric horizontal gradient estimations (black arrow). Applying the horizontal gradient estimation (white arrow) reduces some of the systematic errors in the solution.

March and the failure of the model in GAMIT, which assumes horizontally homogenous stratified atmosphere with the simple gradient, although the realistic atmosphere is horizontal homogenous stratified atmosphere with the waved disturbance.

The strong jet flows associated with cold fronts as stud- ied in this paper are frequently found in spring and autumn in Japanese Islands. Among the GPS permanent array (GEONET) composed of about 1000 domestic sites in Japan by the Geographical Survey Institute (GSI), many sites are located in mountainous regions because of the steep topography commonly found in the island arcs in the Western Pacific subduction zones. Thus the type of phenomena reported here could frequently occur and cause the systematic errors of the horizontal site positions. Our study shows that accounting for horizontal gradients caused by mountain lee waves and other local atmospheric perturbations can not reduce the systematic errors only applying the simple atmospheric gradient model in GPS monitoring of the crustal movements in the plate boundary zones with the steep topography.

\section{Conclusion}

The GPS meteorological analysis detected asymmetric non-linear atmospheric structure which may suggest atmospheric mountain lee waves excited by a strong westerly wind ahead of an approaching cold front on 7th March 1997, as evidenced by significant atmospheric gradients are detected by GPS data analysis and numerical atmospheric model. According to the analysis, the result from the sites along the east coast of the Izu Peninsula indicates a wet and cold atmosphere to the west of these sites. On the contrary, the island site $6 \mathrm{~km}$ east of the east coast detects a strong gradient to the east of the island. Cloud images of the region indicate rows of the clouds due to mountain lee waves consistent with the GPS measurements. A numerical simulation explains the mountain lee waves. The atmospheric inhomogeneity induces large systematic errors in the site horizontal estimates from the processing without the atmospheric horizontal gradient estimations, and the analysis including the simple gradient perturbed atmospheric model still retains some of the systematic errors. This study assigns that the large errors in position estimates can result from atmospheric phenomena like mountain lee waves in regions with mountainous topography like the Japanese Islands.

Acknowledgments. This study is a part of 'GPS Meteorological Project in Japan' supported by Japanese Ministry of Education, Culture, Sports, Science and Technology. We thank P. Fang for his help to draw the atmospheric gradient map. We also thank K. Takai, F. Kimata and E. Emoto for their suggestion for this study. The manuscript is polished by the critical review by Y. E. Bar-Sever and K. Heki. We use the observed data of GEONET GPS permanent array by GSI.

\section{References}

Aoki, Y., P. Segall, T. Kato, P. Cervelli, and S. Shimada, Imaging magma transport during the 1997 seismic swarm off the Izu Peninsula, Japan, Science, 286, 927-930, 1999.

Bar-Sever, Y. E., P. M. Kroger, and J. A. Borjesson, Estimating horizontal gradients of tropospheric path delay with a single GPS receiver, $J$. Geophys. Res., 103, 5,019-5,035, 1998.

Boucher, C., Z. Altamimi, and P. Sillard, Results and analysis of the ITRF96, IERS Technical Note, 24, 166 pp., 1998.

Chen, G. and T. A. Herring, Effects of atmospheric azimuth asymmetry on the analysis of space geodetic data, J. Geophys. Res., 102, 20,48920,502, 1997.

Geographical Survey Institute, Crustal movements in the Izu Peninsula and its vicinity, Rep. Coord. Commit. Earthquake Prediction, 58, 290-311, 1997. 
Hooke, W. H., Gravity waves, in Mesoscale Meteorology and Forecasting, pp. 272-288, Amer. Meteor. Soc., 1986.

King, R. W. and Y. Bock, Documentation for the GAMIT GPS Analysis Software, Massachusetts Institute of Technology, Cambridge, 2000.

MacMillan, D. S., Atmospheric gradients from very long baseline interferometry observation, Geophys. Res. Lett., 22, 1041-1044, 1995.

Okada, Y. and E. Yamamoto, Dyke intrusion model or the 1989 seismovolcanic activity Off Ito, Central Japan, J. Geophys. Res., 96, 10361-10376, 1991.

Saito, K., Semi-implicit fully compressible version of the MRI mesoscale nonhydrostatic model-Forecast experiment of the 6 August 1993 Kagoshima Torrential Rain_, Geophys. Mag. Ser. 2, 2, 109-137, 1997. Shimada, S., Y. Fujinawa, S. Sekiguchi, S. Ohmi, T. Eguchi, and Y. Okada, Detection of a volcanic fracture opening in Japan using Global Positioning System measurements, Nature, 343, 631-633, 1990.

S. Shimada (e-mail: shimada@bosai.go.jp), H. Seko, H. Nakamura, K. Aonashi, and T. A. Herring 